\title{
Influence of implantation voltage on the biological properties of zinc-implanted titanium
}

\author{
Hongqin Zhu ${ }^{1}$, Guodong $\mathrm{Jin}^{1}$, Huiliang Cao, Yuqin Qiao, Xuanyong Liu*
}

State Key Laboratory of High Performance Ceramics and Superfine Microstructure,

Shanghai Institute of Ceramics, Chinese Academy of Sciences, Shanghai 200050,

People's Republic of China

\section{*Corresponding Author:}

Prof. Xuanyong Liu

State Key Laboratory of High Performance Ceramics and Superfine Microstructure, Shanghai Institute of Ceramics, Chinese Academy of Sciences, Shanghai 200050, China

E-mail: xyliu@mail.sic.ac.cn

Tel: 86-21-52412409

${ }^{1}$ These authors contributed equally to this work 


\begin{abstract}
Titanium (Ti) has been widely used as orthopedic and dental implants, it is very important to improve the osteogenic activity and antibacterial ability of titanium implants. The objective of this work was to investigate the osteogenic and antibacterial ability of the zinc $(\mathrm{Zn})$ ion implanted $\mathrm{Ti}$ at different implantation voltage. X-ray photoelectron spectroscopy results indicate that $\mathrm{Zn}$ was successfully implanted into titanium. Zn-implanted titanium surfaces exhibit enhanced cell proliferation ability, alkaline phosphatase (ALP) activity, and partial resistance to both Escherichia coli (E.coli) and Staphylococcus aureus (S. aureus). The excellent osteogenic and antibacterial ability are highly related to the implantation voltage. The cell proliferation and antibacterial ability can be enhanced by increasing the implantation voltage from $15 \mathrm{kV}$ to $30 \mathrm{kV}$, indicating that $\mathrm{Zn}$ implantation at $30 \mathrm{kV}$ is more beneficial to both osteogenic and antibacterial ability.
\end{abstract}

Keywords: zinc; titanium; ion implantation; osteogenic activity; antibacterial ability 


\section{Introduction}

Titanium (Ti) and its alloys which possess excellent mechanical properties, good corrosion resistance and outstanding biocompatibility are widely used as standard orthopedic and dental implants [1]. Despite the fact that Ti implants have achieved a high success rate clinically, their osseointegration and antibacterial ability are inadequate, implant failures still occur owing to implant-associated bacterial infections and remain one of the intractable complications [2]. Therefore, surface modification of Ti implants which is effective and simple is urgently needed to improve both osteogenic and antibacterial ability simultaneously.

In recent years, Ag ion implantation has been considered as an important technology to endow biomaterials with specific antibacterial effect [3-5]. However, $\mathrm{Ag}$ is a toxic element, which may be restricted in the application of biomaterials, considering the stringent safety requirement for biomaterials. As an essential trace element, zinc ( $\mathrm{Zn})$ has been widely reported to have the ability to enhance osteogenetic function [6], promote cell proliferation, alkaline phosphatase (ALP) activity and osteoblast marker gene expressions [7, 8]. In addition, Zn ions show antibacterial ability on various kinds of bacteria including both Gram negative and Gram positive bacteria at appropriate concentrations [9-11]. Our previous study demonstrated that titanium after $\mathrm{Zn}$ ion implantation for various times at the same implantation voltage can enhance the proliferation of osteoblasts, stimulate the initial adhesion and spreading activity, alkaline phosphate activity of rat bone mesenchymal stem cells (rBMSCs) [12]. It was also observed that Zn-implanted titanium exhibited 
partly antibacterial ability on both Escherichia coli (E. coli) and Staphylococcus aureus (S. aureus).

Plasma immersion ion implantation (PIII) technology is a non-line-of-light process, which has been widely used for the surface modification of various biomaterials in recent years [1]. PIII technique has been demonstrated to enhance the osteogenic and antibacterial ability of various kinds of biomaterials $[8,13]$. In the present work, $\mathrm{Zn}$ was introduced into titanium surface using plasma immersion ion implantation (PIII) at different implantation voltage for $1.5 \mathrm{~h}$ to achieve both osteogenic and antibacterial ability. The influences of implantation voltage on the behaviors of osteoblasts, rBMSCs, and bacteria were investigated.

\section{Experimental details}

2.1. Plasma immersion ion implantation (PIII)

Titanium plates $(10 \mathrm{~mm} \times 10 \mathrm{~mm} \times 1 \mathrm{~mm})$ were ultrasonically cleaned in acetone, ethanol and deionized water successively, followed by $5 \mathrm{wt} \%$ oxalic acid etching at $100{ }^{\circ} \mathrm{C}$ for 2 hours and ultrasonically cleaned in deionized water for $30 \mathrm{~min}$. The titanium plates were cleaned by radio frequency (RF) argon ions for $15 \mathrm{~min}$ at a bias of $-550 \mathrm{~V}$ before zinc ion implantation. The pulsed high voltage and arc current were synchronized at a pulsing frequency of $5 \mathrm{~Hz}$. A zinc cathodic arc was utilized as the plasma source and zinc ions were implanted into titanium surface at $15 \mathrm{kV}$ and 30 $\mathrm{kV}$ of implantation voltage for $1.5 \mathrm{~h}$ (Represented as $\mathrm{Zn} 15$ and $\mathrm{Zn} 30$, respectively). During ion implantation, the sample stage was cooled by circulating water in order to 
keep the specimens temperature at nearly $25^{\circ} \mathrm{C}$.

\subsection{Surface characterization}

\subsubsection{Surface structure and chemistry}

The surface morphologies of titanium before and after $\mathrm{Zn}$ ion implantation were examined by scanning electron microscopy (SEM) (S-3400, Japan). The chemical states and zinc elemental depth profiles were measured by X-ray photoelectron spectroscopy (XPS, Physical electronics PHI 5802).

\subsubsection{Dynamic potential polarization test}

The dynamic potential polarization tests were acquired in $0.9 \% \mathrm{NaCl}$ solution (at a $\mathrm{pH}$ of 7) using a CHI760C electrochemical workstation (CHI Instruments, Inc. Shanghai).

\subsection{Cell proliferation assay}

The proliferation ability of mouse osteoblastic MC3T3-E1 cells was determined using the alamarBlue ${ }^{\mathrm{TM}}$ assay (AbDSerotec Ltd, UK). Three samples were examined for each incubation period (1, 4, 7 days). After each incubation time, the culture medium was removed and $0.5 \mathrm{ml}$ of fresh medium with $10 \%$ alamarBlue ${ }^{\mathrm{TM}}$ was added, the samples with the cells were incubated for another $4 \mathrm{~h}$. Afterwards $100 \mu \mathrm{l}$ of the medium were transferred to a 96-well plate (Nunc, USA). Accumulation of reduced alamarBlue $^{\mathrm{TM}}$ in the medium was tested using an enzyme labeling instrument (BIO 
TEK, ELX 800), at extinction wavelengths of $570 \mathrm{~nm}$ and $600 \mathrm{~nm}$. Calculation of proliferation rate followed the instruction of the alamarBlue ${ }^{\mathrm{TM}}$ assay.

After each incubation period, the specimens with the MC3T3-E1 cells were washed with PBS thrice and fixed with $2.5 \%$ glutaraldehyde. Before SEM examination, the specimens were dehydrated in gradient ethanol solutions $(30,50,75$, 90, 95 and $100 \mathrm{v} / \mathrm{v} \%$ ) for $10 \mathrm{~min}$ each sequentially, followed by drying in the hexamethyldisilazane ethanol solution series.

\subsection{Alkaline phosphate activity assay}

Rat BMSCs (Stem Cell Bank, Chinese Academy of Sciences, Shanghai, China) were seeded on the various surfaces to evaluate the ALP activity. After culturing for 7 and 14 days, the specimens with the cells were rinsed with PBS twice and fixed with 4\% paraformaldehyde (PFA) solution (Sigma, USA), followed by incubating in a mixture of naphtol AS-MX phosphate and fast blue RR salt for ALP staining. For the quantitative analysis, the cells were incubated with p-nitrophenyl phosphate (pNPP) (Sigma, USA) at $37{ }^{\circ} \mathrm{C}$ for $0.5 \mathrm{~h}$. The ALP activity was determined by measuring the absorbance at a wavelength of $405 \mathrm{~nm}$, and the ALP levels were normalized to the total protein content, which was determined by the BCA protein assay.

\subsection{Antibacterial assay}

The antibacterial ability of the concerned specimens was evaluated using both $E$. coli (ATCC 25922) and S. aureus (ATCC 25923). The samples were sterilized in $75 \%$ 
ethanol for 2 h. $60 \mathrm{ul}$ suspension with a bacterial concentration of $10^{7} \mathrm{cfu} / \mathrm{ml}$ was dripped on each sample surface. After incubation at $37{ }^{\circ} \mathrm{C}$ for $24 \mathrm{~h}$, the dissociated suspension was collected and introduced into an agar culture plate for further incubation for $24 \mathrm{~h}$. The active bacteria were counted in accordance with the National Standard of China GB/T 4789.2 protocol and the antibacterial ratio was calculated as follows:

$$
\frac{(A-B)}{A} \times 100 \%
$$

where $\mathrm{A}$ is the number of bacteria on the control sample (CFU/sample) and $\mathrm{B}$ is the number of bacteria on the testing specimens (CFU/sample).

In the SEM test, a suspension with a concentration of $10^{7} \mathrm{cfu} / \mathrm{ml}$ was dripped onto the various surfaces to a density of $60 \mu \mathrm{l} / \mathrm{cm}^{2}$, after incubation at $37{ }^{\circ} \mathrm{C}$ for $24 \mathrm{~h}$, fixed with $2.5 \%$ glutaraldehyde, and dehydrated according to the same procedures used in Section 2.3.

\subsection{Statistical analysis}

All the data were expressed as means \pm standard deviations. Statistically significant differences $(\mathrm{P})$ were determined using two-way analysis of variance, $\mathrm{P}$ values $<0.05$ were considered statistically significant. Statistical analysis was done by a GraphPad Prism statistical software package.

\section{Results}

\subsection{Surface characteristics}


Figure 1a-c display the surface morphologies of the oxalic acid etched titanium and Zn-implanted titanium. A micro pitted rough structure with clearly visible grain boundaries can be obviously observed on the surfaces of oxalic acid etched titanium. Importantly, the surface structures still remain the same after $\mathrm{Zn}$ ion implantation, both at $15 \mathrm{kV}$ and $30 \mathrm{kV}$.

To evaluate the elemental depth profiles and chemical states, both $\mathrm{Zn} 15$ and Zn30 were analyzed by XPS and the results are shown in Fig. 1d-f. The Zn elemental depth profiles are presented in Fig. 1d, it is obvious that depth profiles of $\mathrm{Zn}$ in both $\mathrm{Zn} 15$ and $\mathrm{Zn} 30$ resemble Gaussian distributions, while the high content of $\mathrm{Zn}$ near the surface arises from deposition of $\mathrm{Zn}$ during PIII. Interestingly, the peak content of $\mathrm{Zn}$ in $\mathrm{Zn} 30$ is significantly higher than that of $\mathrm{Zn} 15$ and moves to the inside, this is ascribed to the fact that the energy of the ionized $\mathrm{Zn}$ ions in $\mathrm{Zn} 30$ is much higher than that of $\mathrm{Zn} 15$.

Figure 1e shows the $\mathrm{Zn} 2 \mathrm{p}$ spectra obtained from the surface and interior of $\mathrm{Zn} 15$, the peaks located at $1,021.5 \mathrm{eV}$ and $1,020.2 \mathrm{eV}$ correspond to zinc oxide $(\mathrm{ZnO})$ and metallic zinc respectively [14]. The $\mathrm{Zn} 2 \mathrm{p}$ spectra obtained from $\mathrm{Zn} 30$ also suggests that $\mathrm{Zn}$ element exists as $\mathrm{ZnO}$ at surface while in the form of metallic $\mathrm{Zn}$ in the interior (Fig. 1f).

To investigate the corrosion resistance of the various samples, potentiodynamic polarization tests were performed in a $0.9 \% \mathrm{NaCl}$ solution and the results are presented in Fig. 2a. It is evident that the corrosion potential $\left(\mathrm{E}_{\mathrm{cor}}\right)$ of $\mathrm{Zn} 15$ shifts negatively comparing to $\mathrm{Ti}$, and a more markedly shift can be observed from $\mathrm{Zn} 30$, 
indicating that the corrosion resistance decreases with the increase of implantation voltage. This is likely due to the lattice defects in the near surface, which were generated during plasma immersion ion implantation process, provide fast diffusion paths for $\mathrm{Cl}^{-}$from $0.9 \% \mathrm{NaCl}$ solution and increase corrosion [13]. These lattice defects were more serious with the increase of implantation voltage, thus generating more fast diffusion paths.

\subsection{Response of MC3T3-E1 cells}

The MC3T3-E1 cells cultured on the various surfaces for 1, 4, and 7 days are shown in Fig. 3a. After culturing for 1 day, although some of the cells exhibit round morphologies on the titanium surface, most of the cells spread well with filopodia, while the cells on the Zn15 and Zn30 spread better with a large number of filopodia. After culturing for 4 days, clustered MC3T3-E1 cells cover the majority of the sample surfaces with connections through filopodia, especially on Zn30. The cells completely cover the sample surfaces after incubation for 7 days.

The proliferation of MC3T3-E1 cells was evaluated using alamarBlue ${ }^{\mathrm{TM}}$ assay, the results are shown in Fig. 3b. Cell proliferation on Zn30 is slightly higher than those on Ti and Zn15 after incubation for 1 day. However, after culturing for 4 days, the cells on $\mathrm{Zn} 15$ exhibits slightly higher proliferation rate than $\mathrm{Ti}$, whereas $\mathrm{Zn} 30$ shows the highest cell proliferation. This trend becomes much more obvious after incubation for 7 days, indicating that $\mathrm{Zn}$-implanted titanium surfaces are favorable for 
the proliferation of MC3T3-E1 cells, and cell proliferation increases with increasing implantation voltage.

\subsection{Response of rBMSCs}

The rBMSCs cultured on Ti, Zn15, and Zn30 for 7 and 14 days were stained with the ALP kit and the results are shown in Fig. 3c. The ALP-positive areas on Zn15 are larger than that on Ti after culturing for 7 days, however, Zn30 exhibits similar areas as Ti. After culturing for 14 days, the ALP-positive areas on Zn15 and Zn30 are comparable and are larger than that on Ti. These results were confirmed by the quantitative analysis shown in Fig. 3d, suggesting that $\mathrm{Zn}$ ion implantation at different voltages can stimulate the differentiation of rBMSCs.

\subsection{Response of bacterial cells}

To evaluate the antibacterial ability of $\mathrm{Ti}, \mathrm{Zn} 15$, and $\mathrm{Zn} 30$, bacterial counting method was used. Fig. 4a shows the typical photographs of bacteria colonies on agar plates. The amounts of E.coli on Zn15 and Zn30 are reduced by approximately $26 \%$ and $40 \%$, respectively. The reductions of $S$. aureus on $\mathrm{Zn} 15$ and $\mathrm{Zn} 30$ are approximately $24 \%$ and $41 \%$, respectively disclosing a similar trend, indicating that Zn-implanted titanium shows partly antibacterial ability on both E. coli and S. aureus and the antibacterial ability increases with the increase of implantation voltage.

The morphologies of both E. coli and S. aureus are examined by SEM. Fig. 4c shows the typical rod-shaped E. coli seeded on titanium, the bacteria on $\mathrm{Zn} 15$ look 
corrugated and the number of which is much less. It is interesting to mention that some of the E. coli on Zn30 lose the typical rod shape and become flat with serrated edges in contact with the surface, indicating that Zn30 has the best antibacterial ability. Similarly, the $S$. aureus bacterial cells exhibit spherical shapes with smooth surfaces on titanium, however, the number of them become less on $\mathrm{Zn} 15$ and $\mathrm{Zn} 30$, especially on Zn30, indicating that Zn30 exhibits best antibacterial ability against $S$. aureus.

\section{Discussion}

Implants that possess both osteogenic and antibacterial ability are urgently needed in order to improve the success rate of biomedical devices. Attempts have been made but the methods used are complicated and costly. Here we reported an effective and simple method using plasma immersion ion implantation (PIII) to introduce $\mathrm{Zn}$ into titanium surface to achieve good osteogenic and partly antibacterial ability. Our previous study indicated that Zn-PIII at $15 \mathrm{kV}$ for various times can stimulate the proliferation of osteoblasts and differentiation of rBMSCs [12]. Herein, $\mathrm{Zn}$ was introduced into titanium surface at different voltage for $1.5 \mathrm{~h}$, the proliferation of osteoblasts can be enhanced (Fig. 3b), the osteogenic differentiation of rBMSCs is improved (Fig. 3d), additionally, Zn-implanted titanium exhibits partly antibacterial effect (Fig. 4).

As we all know, the standard electrode potential of the titanium oxide, which forms spontaneously on the surface of $\mathrm{Ti}$ when exposed in air, that is $\mathrm{E}^{\circ} \mathrm{TiO} 2=-0.502$ $\mathrm{V}$ is markedly more positive than that of $\mathrm{Zn}, \mathrm{E}^{\circ} \mathrm{Zn}=-0.7618 \mathrm{~V}$ [15]. According to the 
electrochemical theory, a micro-galvanic couple is likely formed between the implanted $\mathrm{Zn}$ and $\mathrm{Ti}$ when $\mathrm{Zn}$-implanted samples were placed in aqueous solution, this will increase the corrosion of samples and release $\mathrm{Zn}^{2+}$ ions to the microenvironment between the samples and cells. On the other hand, the $\mathrm{Zn}$ depth profile of Zn30 moves to the inside compared to Zn15 (Fig. 1d), thus resulting in the release of $\mathrm{Zn}^{2+}$ ions becomes much more difficult. The cumulative $\mathrm{Zn}^{2+}$ ions leached from $\mathrm{Zn} 30$ is lower than that from $\mathrm{Zn} 15$ from 7 to 21 days (Fig. 2b). It is accepted that the influence of $\mathrm{Zn}$ on the differentiation of mesenchymal stem cells is dose dependent $[16,17]$. The better osteogenic and antibacterial ability of Zn30 compared to $\mathrm{Zn} 15$ may be ascribed to the appropriate dose of local $\mathrm{Zn}^{2+}$ ions at the interface of the biomaterials [18]. On the other hand, the higher $\mathrm{Zn}$ content of $\mathrm{Zn} 30$ at the near surface (45\%) may also generate superior osteogenic activity and antibacterial effect.

\section{Conclusions}

Zinc was successfully introduced into titanium using plasma immersion ion implantation technology. Zn-implanted titanium exhibited enhanced osteogenic and antibacterial effect on both E. coli and S. aureus. The stimulation of cell proliferation and antibacterial ability could be increased by increasing the implantation voltage from $15 \mathrm{kV}$ to $30 \mathrm{kV}$. The excellent osteogenic and antibacterial ability is highly related to the release of $\mathrm{Zn}^{2+}$ ions from the micro-galvanic couple formed between the implanted $\mathrm{Zn}$ and Ti. 


\section{Acknowledgements}

Financial support from the National Science Foundation for Distinguished Young

Scholars of China (51525207), National Natural Science Foundation of China (31570973), Shanghai Committee of Science and Technology, China (15441904900, 14XD1403900), Shanghai Rising-Star Program (15QA1404100), and Youth Innovation Promotion Association CAS (2015204) are acknowledged.

\section{References}

[1] X. Liu, P.K. Chu, C. Ding, Surface modification of titanium, titanium alloys, and related materials for biomedical applications, Mat. Sci. Eng. R, 47 (2004) 49-121.

[2] S.B. Goodman, Z. Yao, M. Keeney, F. Yang, The future of biologic coatings for orthopaedic implants, Biomaterials, 34 (2013) 3174-3183.

[3] X.H. Xiao, W. Dong, W. Wu, T.C. Peng, X.D. Zhou, F. Ren, C.Z. Jiang, Antibacterial Silver-Containing Silica Glass Prepared by Ion Implantation, J. Nanosci Nanotechno., 10 (2010) 6424-6427.

[4] W.Q. Li, X.Q. Liu, Y.Q. Wang, Z.G. Dai, W. Wu, L. Cheng, Y.P. Zhang, Q. Liu, X.H. Xiao, C.Z. Jiang, Design of high-performance memristor cell using W-implanted $\mathrm{SiO}_{2}$ films, Appl. Phys. Lett., 108 (2016). [5] X.Y. Song, Z.G. Dai, X.H. Xiao, W.Q. Li, X.D. Zheng, X.Z. Shang, X.L. Zhang, G.X. Cai, W. Wu, F.L. Meng, C.Z. Jiang, Formation of Carbonized Polystyrene Sphere/hemisphere Shell Arrays by Ion Beam Irradiation and Subsequent Annealing or Chloroform Treatment, Sci. Rep., 5 (2015).

[6] H.-J. Seo, Y.-E. Cho, T. Kim, H.-I. Shin, I.-S. Kwun, Zinc may increase bone formation through stimulating cell proliferation, alkaline phosphatase activity and collagen synthesis in osteoblastic MC3T3-E1 cells, Nutr. Res. Pract., 4 (2010) 356-361.

[7] K. Yusa, O. Yamamoto, M. Fukuda, S. Koyota, Y. Koizumi, T. Sugiyama, In vitro prominent bone regeneration by release zinc ion from Zn-modified implant, Biochem. Bioph. Res Commun., 412 (2011) 273-278.

[8] G. Jin, H. Qin, H. Cao, S. Qian, Y. Zhao, X. Peng, X. Zhang, X. Liu, P.K. Chu, Synergistic effects of dual $\mathrm{Zn} / \mathrm{Ag}$ ion implantation in osteogenic activity and antibacterial ability of titanium, Biomaterials, 35 (2014) 7699-7713.

[9] G. Applerot, A. Lipovsky, R. Dror, N. Perkas, Y. Nitzan, R. Lubart, A. Gedanken, Enhanced antibacterial activity of nanocrystalline $\mathrm{ZnO}$ due to increased ROS - mediated cell injury, Adv. Funct. Mater., 19 (2009) 842-852.

[10] J. Xu, G. Ding, J. Li, S. Yang, B. Fang, H. Sun, Y. Zhou, Zinc-ion implanted and deposited titanium surfaces reduce adhesion of Streptococccus mutans , Appl. Surf. Sci., 256 (2010) 7540-7544.

[11] H. Hu, W. Zhang, Y. Qiao, X. Jiang, X. Liu, C. Ding, Antibacterial activity and increased bone marrow stem cell functions of $\mathrm{Zn}$-incorporated $\mathrm{TiO}_{2}$ coatings on titanium, Acta Biomater., 8 (2012) 904-915.

[12] G. Jin, H. Cao, Y. Qiao, F. Meng, H. Zhu, X. Liu, Osteogenic activity and antibacterial effect of zinc 
ion implanted titanium, Colloid. Surface. B, 117 (2014) 158-165.

[13] H. Cao, X. Liu, F. Meng, P.K. Chu, Biological actions of silver nanoparticles embedded in titanium controlled by micro-galvanic effects, Biomaterials, 32 (2011) 693-705.

[14] V.I. Nefedov, M.N. Firsov, I.S. Shaplygin, Electronic structures of $\mathrm{MRhO}_{2}, \mathrm{MRh}_{2} \mathrm{O}_{4}, \mathrm{RhMO}_{4}$ and $\mathrm{Rh}_{2} \mathrm{MO}_{6}$ on the basis of X-ray spectroscopy and ESCA data, J. Electron Spectrosc., 26 (1982) 65-78.

[15] P. Vanysek, Electrochemical series, CRC Handbook of Chemistry and Physics. CRC press, Boca Raton, DOI (1998).

[16] T. Wang, J.-C. Zhang, Y. Chen, P.-G. Xiao, M.-S. Yang, Effect of zinc ion on the osteogenic and adipogenic differentiation of mouse primary bone marrow stromal cells and the adipocytic trans-differentiation of mouse primary osteoblasts, J. Trace Elem. Med. Bio., 21 (2007) 84-91.

[17] A. Hoppe, N.S. Guldal, A.R. Boccaccini, A review of the biological response to ionic dissolution products from bioactive glasses and glass-ceramics, Biomaterials, 32 (2011) 2757-2774.

[18] Y. Qiao, W. Zhang, P. Tian, F. Meng, H. Zhu, X. Jiang, X. Liu, P.K. Chu, Stimulation of bone growth following zinc incorporation into biomaterials, Biomaterials, 35 (2014) 6882-6897. 


\section{Figure captions}

Fig. 1. Surface morphologies of the oxalic acid etched titanium before and after $\mathrm{Zn}$ ion implantation: (a) As-etched Ti, (b) Zn15, (c) Zn30; (d) Zn depth profiles of Zn15 and $\mathrm{Zn30}$; (e) high resolution spectra obtained from the surface of $\mathrm{Zn} 15$; (f) high resolution spectra obtained from the surface of $\mathrm{Zn} 30$.

Fig. 2. (a) Potentiodynamic polarization curves, (b) $\mathrm{Zn}$ concentrations in $0.9 \% \mathrm{NaCl}$ solution after immersion for 7,14 , and 21 days.

Fig. 3. (a) SEM morphology and (b) reduction percentage of alamarBlue ${ }^{\mathrm{TM}}$ for MC3T3-E1 cells cultured on various surfaces for 1, 4, 7 days; (c) ALP positive areas of rBMSCs cultured on various surfaces for 7 and 14 days and (d) Corresponding colorimetrically qualitative results; ${ }^{*} \mathrm{P}<0.05,{ }^{* *} \mathrm{P}<0.01,{ }^{* * *} \mathrm{P}<0.001$.

Fig. 4. (a) Re-cultivated bacterial colonies on agar with the bacteria concentration being $10^{7} \mathrm{cfu} / \mathrm{ml}$, (b) Percentage reductions of E. coli and S. aureus re-cultured on agar, which were previous dissociated off the various surfaces, with the re-cultivated bacteria concentration being $10^{7} \mathrm{cfu} / \mathrm{ml}$, (c) SEM morphology of E. coli and S. aureus seeded on various surfaces; ${ }^{*} \mathrm{P}<0.05,{ }^{* *} \mathrm{P}<0.01,{ }^{* * *} \mathrm{P}<0.001$. 

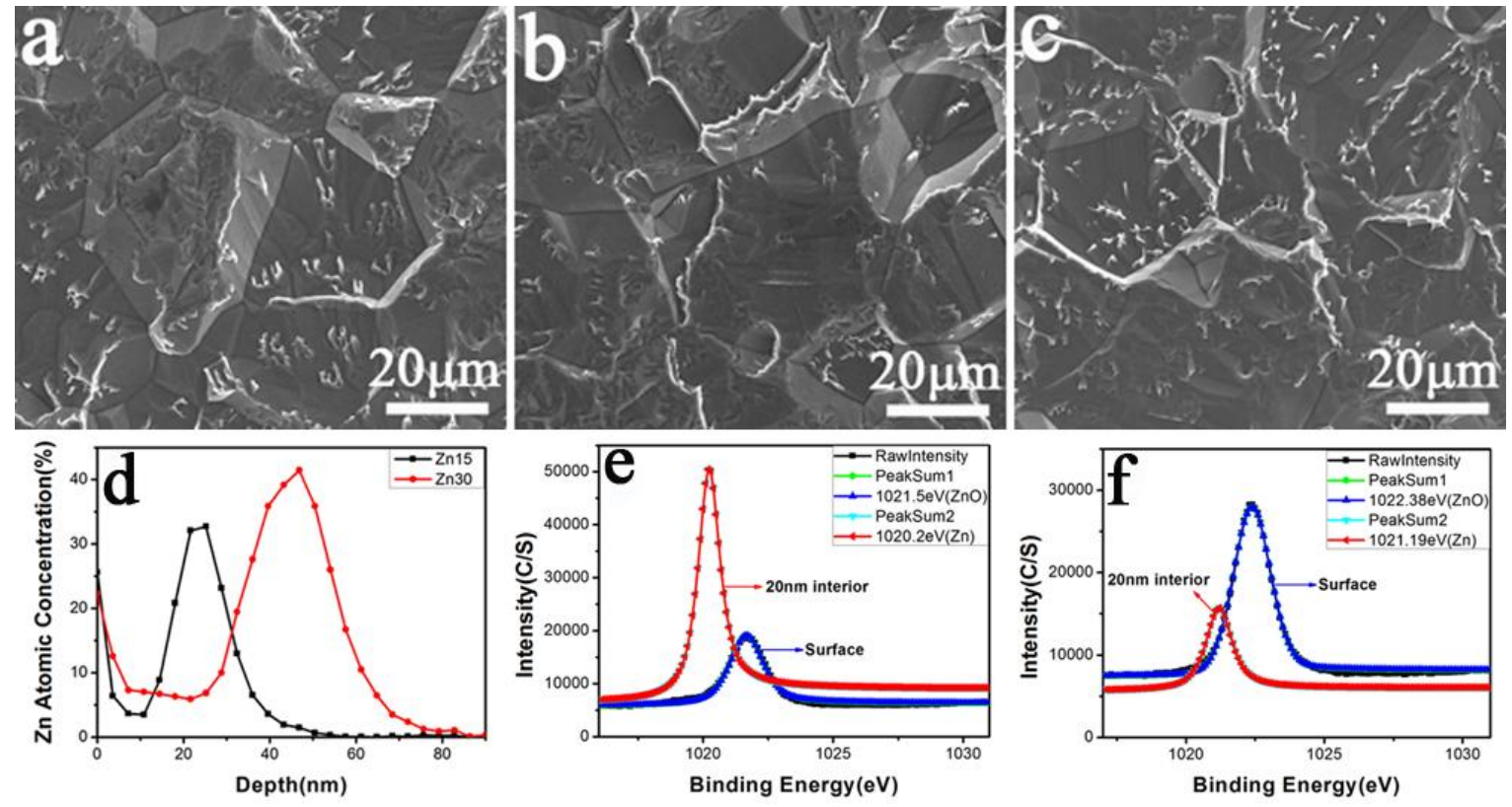

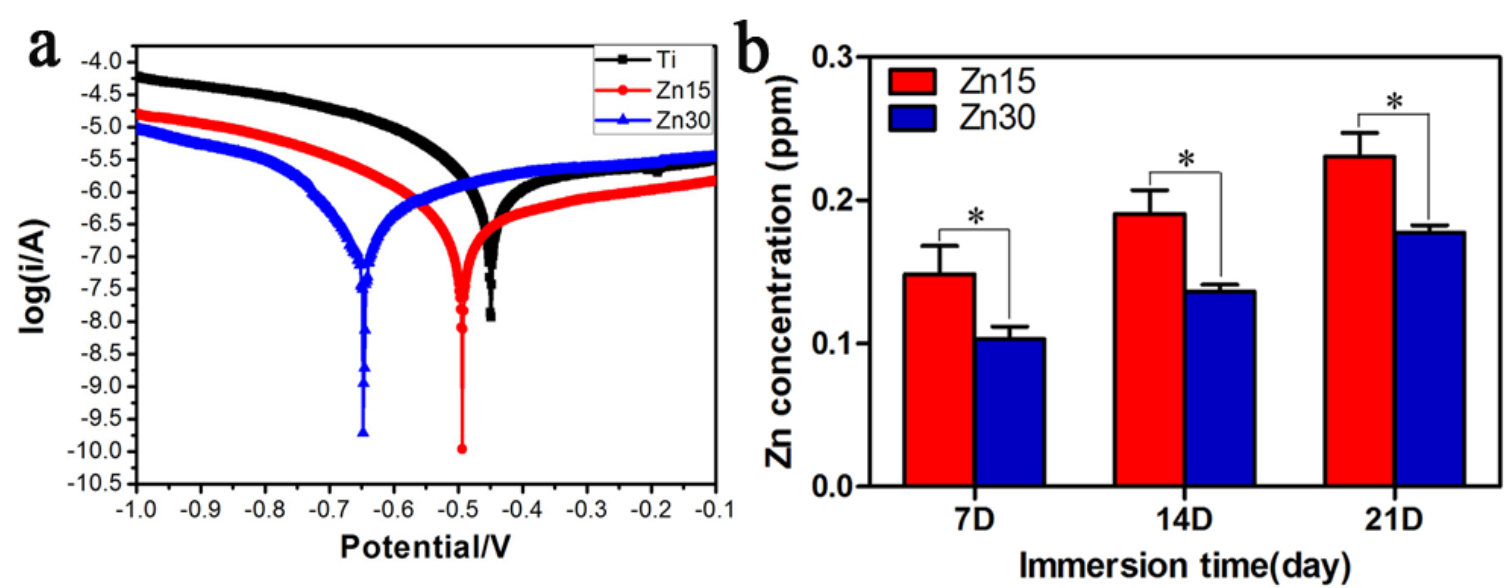

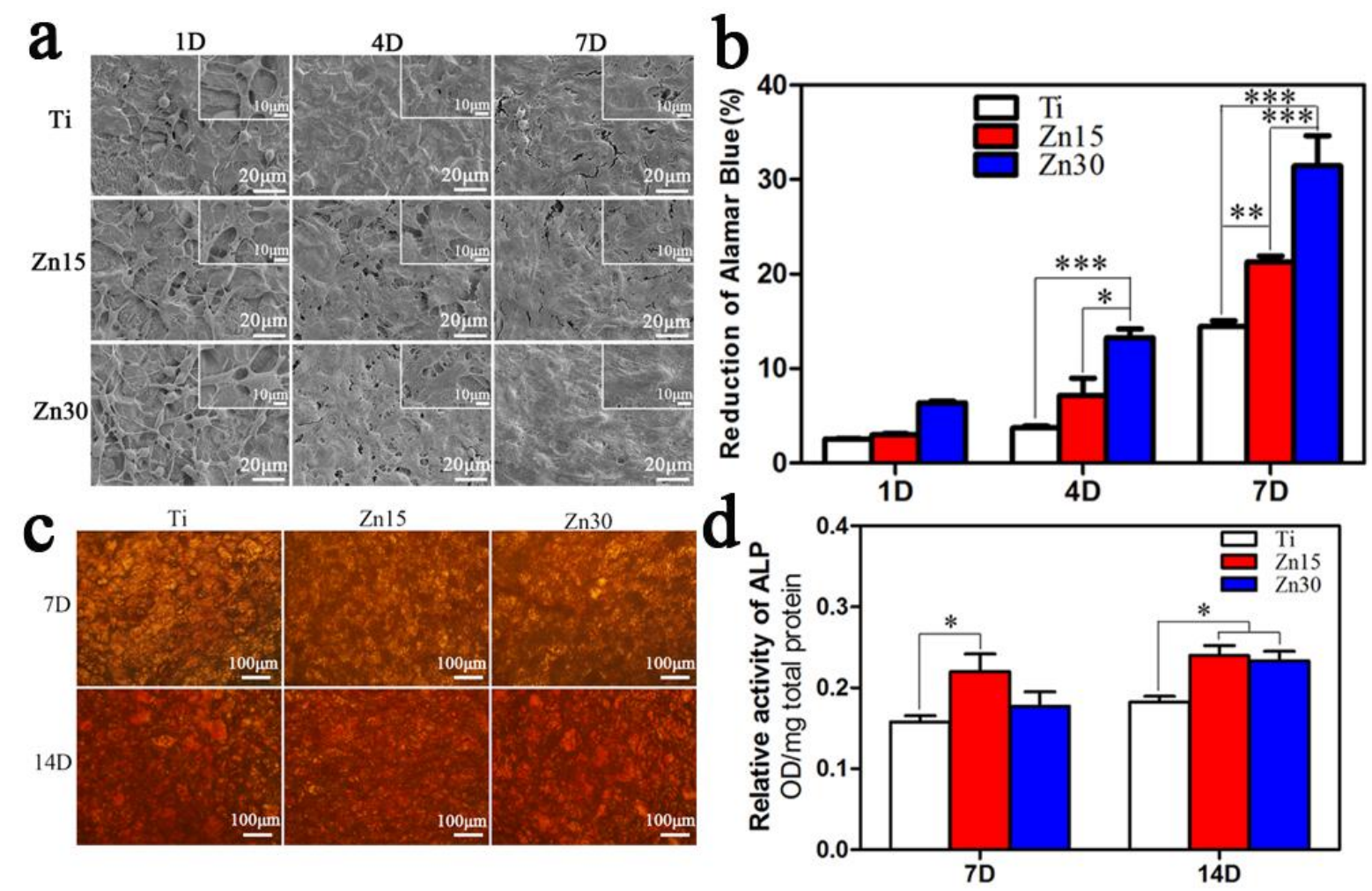

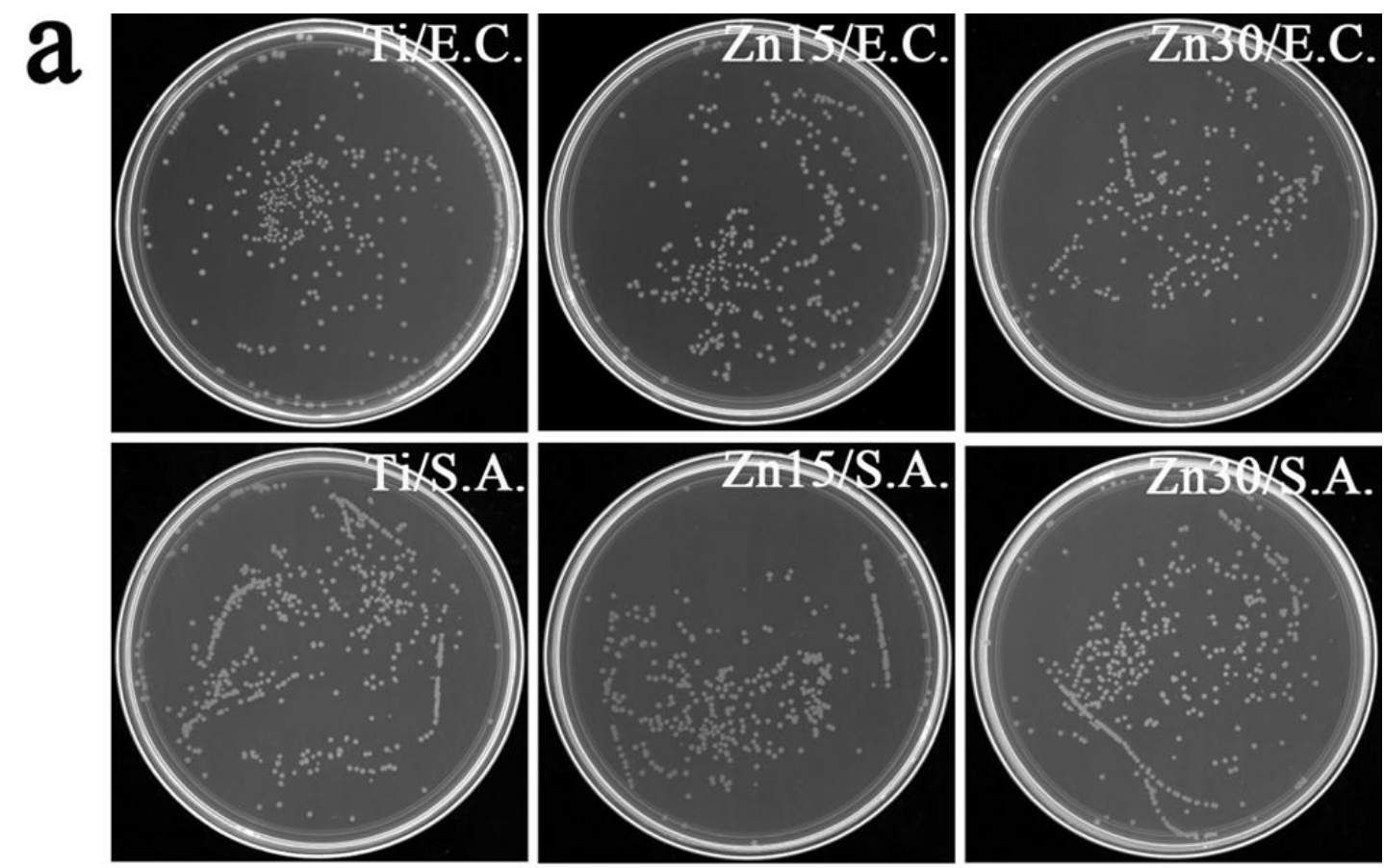

b
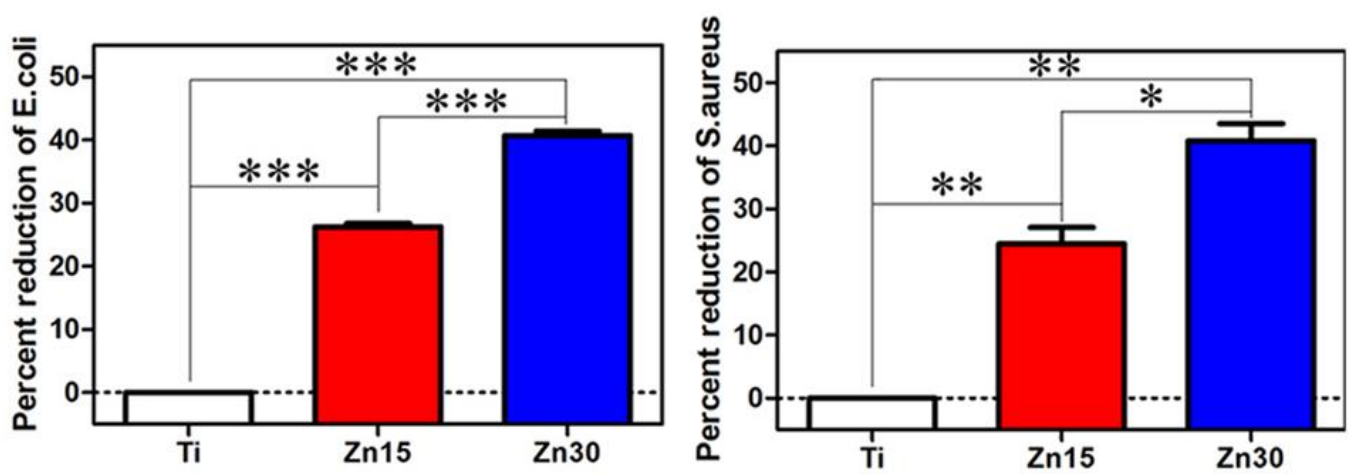

C

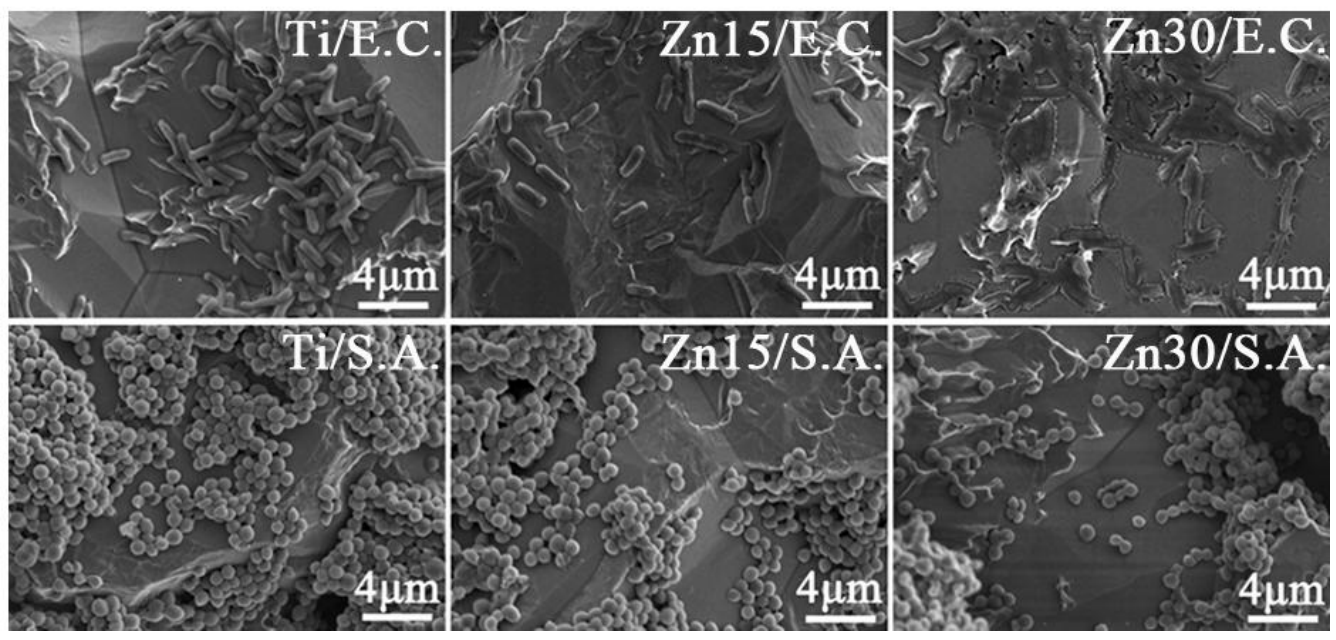

